\title{
Management of isolated distal deep vein thrombosis. A persistent conundrum?
}

\author{
Nalaka Gunawansa, Thilina Gunawardena \\ National Institute of Nephrology and Transplantation, Colombo, Sri Lanka
}

\begin{abstract}
Isolated distal deep vein thrombosis (IDDVT) accounts for approximately 50\% of all patients diagnosed with DVT. While the definitive management of patients with proximal deep vein thrombosis is fairly well defined, IDDVT remains shrouded uncertainty. The great majority of patients with IDDVT may remain with little or no symptoms and have spontaneous resolution of the thrombi. However, a small but significant fraction may show proximal thrombus extension and may proceed to cause pulmonary embolism or late deep venous reflux. Identification of this subgroup of patients with IDDVT who have a greater propensity for thrombus extension or further sequelae remains the cornerstone of individualized management for optimal results.
\end{abstract}

Key words: deep vein thrombosis, pulmonary embolism, calf vein thrombus, venous thromboembolism

Acta Angiol 2020; 26, 2: 65-7I

\section{Introduction}

Systemic anticoagulation remains the standard of care in managing proximal deep vein thrombosis (PDVT). However, the optimal management of isolated distal deep vein thrombosis (IDDVT) remains heavily debated and controversial. IDDVT refers to the deep venous thrombi occurring in the veins distal to and not involving the popliteal vein. These include the tibial and peroneal veins as well as the intramuscular venous plexus of soleus and gastrocnemius muscles. At present, there is no clear consensus among clinicians regarding the clinical significance nor the optimal management of IDDVT. While some argue the probability of proximal propagation, risk of pulmonary embolism (PE) and late post-thrombotic syndrome (PTS) in untreated IDDVT, others argue that it is primarily a self-limiting benign disease and routine treatment is 'overkill'. Hence, an informed precise decision needs to be made regarding the management of IDDVT on an individual basis. This requires balancing the benefits of avoiding short and long-term sequelae by anti-coagulation against potential adverse effects of the treatment and economic burden of such treatment. This update will attempt to revisit the said areas of controversy with a look at the available evidence regarding the optimal management of IDDVT.

\section{Epidemiology}

The estimated annual incidence of deep vein thrombosis (DVT), both PDVT and IDDVT, is approximately I in 1000 adults [ $I$ ] and recurs frequently. VTE is a complex (multifactorial. Among the diagnosed patients with DVT and PE, the mean prevalence of IDDVT is estimated to be around $50 \%(20-70 \%)$ [I] and recurs frequently. VTE is a complex (multifactorial. However, this could be a gross underestimate as the majority of patients with IDDVT remain asymptomatic and may not present to hospital, contributing to under-reporting. The wide variability in IDDVT prevalence across the literature is attributed to the lack of consensus on reporting the patient cohort (symptomatic vs asymptomatic and in-patient vs out-patient). There is further discordance in the modality of diagnostic imaging (duplex ultra-sound — DUS vs venography) used in the diagnosis of IDDVT. 
The immediate danger of DVT is the risk of propagation and PE. Therefore, the primary debate regarding management of IDDVT revolves around the possibility and prevention of PE and associated other complications. According to the Center for Disease Control $(C D C)$ data in the United States, reviewed in 2020, the annual estimated deaths due to PE range between 60,000 to 100,000 [2]. Accordingly, PE is seen as a bigger contributor to annual mortality than motor accidents, breast carcinoma and HIV combined. Wei et al. [3] studied the prevalence of IDDVT among hospitalized patients with diagnosed DVT and PE (collectively referred to as venous thromboembolism; VTE). They reported an IDDVT prevalence of $25 \%$ among all patients with diagnosed $\mathrm{PE}$. In an earlier publication, Mattos et al. [4], reported an IDDVT prevalence of $45 \%$ among outpatients and $27 \%$ among in-patients with confirmed VTE. According to a comprehensive study on DUS based DVT diagnoses, Sapp et al. [5] reported that simultaneous calf vein DVT is found in over $98 \%$ of patients diagnosed with PDVT. The incidence of true IDDVT was $54 \%$, indicating that unless targeted imaging of the calf veins is performed, over half the patients with DVT would be missed and a false negative report would ensue.

\section{Diagnosis}

Given the high prevalence of IDDVT among patients who require hospitalization for VTE, it is imperative that the diagnostic modality used in VTE can detect IDDVT in the absence of PDVT. However, diagnosis of IDDVT itself poses numerous issues. While the vast majority of patients may remain asymptomatic till proximal thrombus extension, even those who do develop symptoms may have negative test results due to the reduced sensitivity of available diagnostic tools. The Wells prediction rule is globally accepted as a pre-test prediction tool in the diagnosis of both DVT and PE [6]. However, the accuracy of the Wells score in predicting the stand-alone IDDVT sub-population is questionable and is considered more predictive only in the presence of proximal DVT [7]. Sartori et al. [8] found that in IDDVT, the sensitivity of Wells predictive rule was only $47 \%$ with a specificity of only $74 \%$. The corresponding negative and positive predictive values for IDDVT were $91 \%$ and $20 \%$ respectively. Serum D-dimer testing is well established as an excellent predictive tool in the diagnosis of VTE, with a reported sensitivity $>92 \%$ although with a poor specificity of $45 \%$ [9]. However, here again, the usefulness of D-dimer in the diagnosis of IDDVT is limited with a reported sensitivity of only $84 \%$ [8].

DUS is the commonest imaging modality used in the confirmation of DVT. The reported sensitivity of DUS in the diagnosis of IDDVT is significantly lower than that of PDVT. According to a meta-analysis by Goodacre et al. [10], the sensitivities of DUS in diagnosing PDVT and IDDVT were $96 \%$ and $71 \%$ respectively. Another recent meta-analysis by Zhang et al. [I I] actually found the sensitivity of DUS in diagnosing IDDVT to be as low as $43 \%$. This stark contrast in diagnostic sensitivities of available predictive and diagnostic tools for PDVT and IDDVT, underlines the issues inherent to the accurate diagnosis of IDDVT.

When using DUS, two common imaging protocols have been described for the diagnosis of DVT. The first is to perform a limited scan of the proximal veins, targeting the popliteal vein and above, where the diagnostic sensitivity is very high. However, this targeted proximal scan will miss those with IDDVT, that amounts to $20-70 \%$ of all patients with DVT [12]. While those who become positive on the proximal scan are treated as for DVT, the 'high-risk' patients with a negative scan get a repeat scan of the proximal veins after one week. This repeat scan is performed with the intention of picking any IDDVT which may have propagated to the popliteal vein after one week. The rationale behind this protocol of imaging is that the PDVT are picked up in the first scan and any clinically significant IDDVTs that show proximal extension are picked up on the second scan. Any such proximal extension of IDDVT is known to occur within 5-7 days while the others are known to resolve spontaneously, thereby being considered clinically insignificant. The second imaging strategy is to perform a whole lower limb scan (inguinal ligament to ankle) as a single test in an effort to diagnose all DVT including IDDVT. While this second strategy allows for more IDDVTs to be picked up early, doubts exist regarding its relevance in the absence of proximal extension. Furthermore, due to the inherent low sensitivity of DUS in detecting IDDVT, a fair number of such patients are likely to be missed on the whole limb scan. The lack of a clear protocol on DUS for DVT diagnosis has led to misinterpretation and variability in reporting among individual radiologists, literature and institutions [7].

In 2018, A multi-disciplinary consensus meeting was held that included the Society of Radiologists, Society for Vascular Ultrasound and American College of Radiologists, to formulate guidelines on DUS for diagnosis of DVT [13]. Accordingly, a consensus guideline was issued that recommends complete leg scanning from inguinal ligament to ankle including tibial and calf veins with compression at $2 \mathrm{~cm}$ intervals. A consensus was also reached regarding the need and place for follow up DUS after an initial positive or negative scan (Table I).

\section{Natural history and complications}

The study by Sapp et al. [5] mentioned above also contributed to the growing opinion that all DVT originates in 
Table I. Duplex Ultra Sound scan protocol in the presence of suspected IDDVT. Adapted from Needleman et al. [13]

\begin{tabular}{|l|l|}
\hline A. Initial positive whole leg DUS & Recommendation \\
\hline Clinical condition & $\begin{array}{l}\text { Repeat DUS in I week (or earlier if symptoms progress) } \\
\text { Start treatment if propagated to the popliteal vein } \\
\text { If no propagation at I week, repeat scan at 2 weeks } \\
\text { If still no propagation at 2 weeks, no further scan warranted }\end{array}$ \\
\hline IDDVT — untreated & Repeat scan not indicated \\
\hline B. Initial negative whole leg DUS & \\
\hline Clinical condition & Recommendation \\
\hline Persistent/worsening symptoms & Repeat DUS in 5-7 days \\
\hline 'High risk' for DVT (hospitalized, active cancer, post-operative etc.) & $\begin{array}{l}\text { Consider repeat scan unless another viable aetiology for } \\
\text { symptoms has been established }\end{array}$ \\
\hline Incomplete study/technical failure & Repeat scan in 5-7 days \\
\hline Suspected ileo-caval DVT & Specialized imaging (CT/MR venography) \\
\hline
\end{tabular}

CT: computed tomography; MR: magnetic resonance

the calf veins. However, it is also postulated that although it originates in the calf veins, the majority do not present or experience symptoms until the thrombus has already propagated to the popliteal vein or proximally [14] and most probably resolve spontaneously. Thrombi that remain confined to the calf rarely cause leg symptoms or symptomatic pulmonary embolism (PE). This results in a gross underestimate on the prevalence of IDDVT as well as the limitation in the number of studies available that deals with IDDVT alone in terms of its sequelae. Conversely, there is a different school of thought that considers IDDVT as a separate entity and compares it with isolated proximal PDVT.

Two major epidemiological studies have been published that compared the individual risk factors for PDVT and IDDVT; the OPTIMEV study and RIETE registry $[15,16]$ clinical presentation and estimated 3 -month survival for each form of VTE were evaluated. Results: Of 5889 patients, 426 had PE. Both studies described IDDVT associated more with transient risk factors such as recent surgical procedure, immobilization, hospitalization, long-distance travel etc. On the contrary, PDVT was associated more with chronic risk factors such as thrombophilia, malignancy and congestive cardiac failure (Table 2).

The vast majority of IDDVT is thought to resolve spontaneously without ever causing significant symptoms. Although PE with IDDVT in the absence of proximal propagation has been observed, the risk of $\mathrm{PE}$ is dramatically increased only after proximal propagation [17]. This emphasizes the importance of understanding the natural history of IDDVT with possible early rec-
Table 2. Risk factors for proximal extension of calf vein thrombus

\begin{tabular}{l}
$\begin{array}{l}\text { Isolated distal deep vein thrombosis. Risk factors for } \\
\text { proximal extension }\end{array}$ \\
\hline I. Positive D-dimer result \\
\hline 2. Extensive thrombus ( $>5 \mathrm{~cm}$ in length) \\
\hline 3. Involvement of multiple veins \\
\hline 4. Thrombus diameter $>7 \mathrm{~mm}$ \\
\hline 5. Close proximity to the proximal (popliteal) vein \\
\hline 6. Unprovoked DVT \\
\hline 7. Active malignancy \\
\hline 8. Prior history of VTE \\
\hline 9. In-patient status (hospitalized) \\
\hline
\end{tabular}

ognition in an effort to minimize proximal extension. Although the associated risk is significantly lower than with PDVT, the possibility of PE and long-term PTS should always be borne in mind during the assessment of IDDVT.

\section{Proximal extension}

While the vast majority of IDDVT will resolve spontaneously without propagation, proximal extension into the popliteal veins remains the commonest complication. The reported rates of the proximal extension are highly variable due to the heterogeneous nature of study populations, diagnostic methods and treatment plans among study groups. A systematic review by Garry et al. [18] studied over 12 papers that discussed the rate 
of proximal extension of IDDVT to be between 0-35\% (mean 8.9\%). Other studies have previously stated higher rates of proximal extension around $25 \%$, within I-2 weeks of presentation [14]and most probably resolve spontaneously. Thrombi that remain confined to the calf rarely cause leg symptoms or symptomatic PE. Hence, the overall rate of proximal extension is likely to be between I0-20\% of all IDDVT. Furthermore, such proximal extension, if it occurs, is likely to occur within the first $\mathrm{I}-2$ weeks from onset, giving rise to the current practice of DUS surveillance for 2 weeks in those patients who do not undergo anti-coagulation. Identification of the 'high-risk' sub-group (Table 2) who are likely to have proximal extension allows selective ant-coagulation in such patients [19].

\section{Pulmonary embolism}

The afore-mentioned review by Garry et al. looked at over 8 studies that described the risk of PE from IDDVT. The reported rates of $P E$ varied between $0-5.8 \%$ with a mean of $1.4 \%$ and is significantly lower compared to PDVT [18]. There were no reported PE-related deaths in any of the studies. This becomes an important determining factor in the decision regarding treatment of IDDVT, as the sole basis for treating such patients actively as opposed to DUS surveillance is based on the immediate risk of PE. Nevertheless, the fact that a small fraction of these patients can actually have PE without proximal thrombus extension needs to be considered in the final decision regarding treatment.

\section{Recurrent thromboembolism}

Another controversial aspect of the management of IDDVT is the risk of recurrent VTE compared to patients with PDVT. Galanaud et al. [20] compared the risk of recurrent VTE in those with PDVT versus IDDVT, 3 years after discontinuation of anticoagulation. The risk of recurrent DVT was significantly lower in the IDDVT group (5.2\% vs. $2.7 \%$ ), while the corresponding risk of PE was similar in both groups ( $1 \%$ vs. $0.9 \%$ ). A subgroup analysis found that among those with IDDVT, age $>50$ years, unprovoked IDDVT and involvement of $>$ I calf vein were associated with a higher risk of recurrence. In a separate study, Sartori et al. [2I] described that male gender and the presence of coexisting active malignancy were associated with a higher risk of recurrence after IDDVT. Another single-center study that enrolled over 800 patients with a first episode of DVT with a mean follow up of 7.6 years found that IDDVT carried a significantly lower risk of recurrent VTE and death compared to IPDVT [22]. Table 3 summarizes the risk factors for recurrence after IDDVT.
Table 3. Risk factors for VTE recurrence after IDDVT

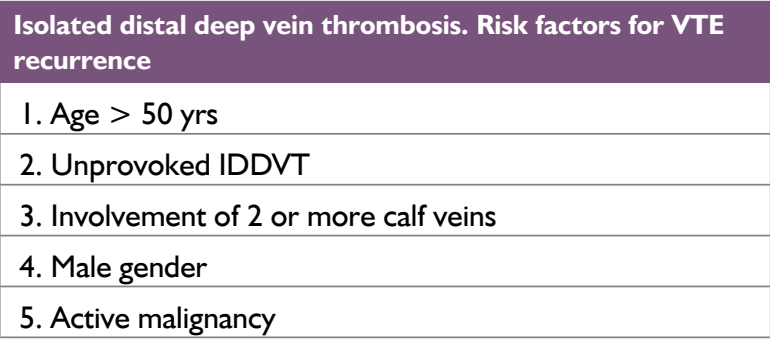

\section{Post-thrombotic syndrome (PTS)}

The risk of PTS after PDVT is estimated to be around $40 \%$ according to the Villata scale [23]. The definitive incidence of PTS after IDDVT is not clearly defined. Available data suggest that patients with IDDVT experience far fewer symptoms of PTS compared to those with PDVT [24, 25]. Meissner et al. [24] reported that at I-year post-diagnosis, $23 \%$ of patients with IDDVT still had symptoms of pain and swelling in the affected leg. This contrasts with PDVT, where up to $54 \%$ are found to be having residual symptoms at I year follow up.

McLafferty et al. [26] studied the long term haemodynamic effects following IDDVT and described the persistence of deep vein reflux in approximately $\mathrm{I} / 3$ of patients after 3.4 years of follow-up. Interestingly, these changes were seen predominantly in proximal venous segments that did not appear to have thrombi in the initial DUS. Hence, they postulated the resulting reflux was caused by previously unseen occult thrombi co-existent with the IDDVT. The CACTUS-PTS study (2020) studied the long-term effects, after 6 years since the first episode of IDDVT. The results showed an overall PTS incidence of $30 \%$, still considerably less than the reported values for patients after PDVT [27].

\section{Management}

\section{Do all patients with IDDVT require treatment?}

While the majority of IDDVT may be self-limiting, the preceding discussion shows that a fair proportion of patients go on to develop significant complications including proximal extension, PE, recurrent VTE and late PTS. The said adverse sequelae are commoner in a subset of patients who carry 'high risk 'characteristics and are left untreated. Hence, a clear distinction needs to be made with regards to the identification of the said 'high-risk' subgroup and initiation of definitive treatment over serial DUS surveillance. While there is no uncertainty with regards to the treatment of PDVT, the decision is often debated in IDDVT due to the potential to do more harm by systemic anti-coagulation. 
The American College of Chest Physicians (ACCP) Consensus Conference in 2008 on the management of DVT failed to distinguish between IDDVT and PDVT, recommending anti-coagulation for a minimum of 3 months for all patients with DVT [28]. The latest ACCP consensus (2016) was more descriptive in its plan for PDVT and IDDVT separately [19]. Accordingly, it recommends identifying the subgroup of patients with IDDVT having 'high-risk' of thrombus extension (Table 2). These patients, as well as, those having severe symptoms are recommended therapeutic anti-coagulation over serial DUS monitoring. Conversely, those patients who do not have high-risk factors for thrombus extension or and are not severely symptomatic can be managed with weekly DUS surveillance. However, both these recommendations were classified grade- $2 \mathrm{C}$ based on a weak evidence base. Hence, the exact therapeutic approach in a given situation with IDDVT remains heavily debated and leaves room for tremendous individual variations in practice.

\section{Anti-coagulation}

Once a decision was made to start anti-coagulation, the recommended duration of therapy was a minimum of 3 months, the same as for PDVT [19]. In the subgroup of patients where therapeutic anti-coagulation is not commenced and are monitored by serial DUS, initiation of treatment is recommended only if there is a proximal extension on repeat imaging. The choice of anti-coagulation agent can be based on individual preference and feasibility ranging from low molecular weight heparin (LMWH), vitamin-K antagonists (warfarin) or one of the novel oral anti-coagulants such as rivaroxaban or apixaban. The use of compression stockings was not routinely recommended in the absence of clear benefit in reducing the incidence of PTS. However, it was stated that stockings may be used on an individual basis for symptom relief [29]singlecentre studies without placebo control. We aimed to assess the efficacy of ECS, compared with placebo stockings, for the prevention of PTS. Methods We did a multicentre randomised placebo-controlled trial of active versus placebo ECS used for 2 years to prevent PTS after a first proximal DVT in centres in Canada and the USA. Patients were randomly assigned to study groups with a web-based randomisation system. Patients presenting with a first symptomatic, proximal DVT were potentially eligible to participate. They were excluded if the use of compression stockings was contraindicated, they had an expected lifespan of less than 6 months, geographical inaccessibility precluded return for follow-up visits, they were unable to apply stockings, or they received thrombolytic therapy for the initial treatment of acute DVT. The primary outcome was PTS diagnosed at
6 months or later using Ginsbergs criteria (leg pain and swelling of $\geq I$ month duration.

\section{What is the optimal duration of anti-coagulation?}

Due to the relatively poor evidence base behind the above ACCP recommendations, individual practices in IDDVT management still vary with no clear consensus. In his publication, Palareti argues that it is impossible to classify patients as asymptomatic IDDVT, as when a patient is either referred to or presents to hospital for DUS to exclude DVT, he/she is invariably symptomatic [30]. He goes on to point out that once IDDVT is diagnosed and the diagnosis is informed to the patient, they are likely to request some form of treatment, at least for symptomatic relief. Hence, in his perspective, he recommends treatment for all patients diagnosed with IDDVT, with possibly a shorter duration (4-6 weeks) for those deemed 'low-risk'. He defines this 'low-risk' subgroup as those having the first episode of DVT, provoked by a reversible risk factor and are not hospitalized or immobilized. This abbreviated 6-week regime has also been recommended by other studies who found it as effective as the standard duration of $I 2$ weeks. Pinede et al. (DOVTAK tria-200 I) [3 I] reported no advantage of a 12-week treatment schedule over the abbreviated 6-week course. Conversely, Ferrara et al. [32] reported a significantly higher rate of proximal thrombus extension with 6 weeks treatment as opposed to 12 weeks, especially among those with 2 or more calf veins involved. A meta-analysis by Franco et al. [33] also found a significantly lower rate of VTE recurrence among those who were treated for 12 weeks as opposed to 6 weeks.

\section{The ongoing debate}

The question of whether all patients diagnosed with IDDVT require anti-coagulant therapy remains one of the biggest conundrums in clinical practice. To date, there appears to be no final solution with conflicting reports from available studies, especially in the 'lowrisk' patient with the first episode of IDDVT. Few prospective randomized studies have assessed the efficacy of anti-coagulation versus serial DUS and selective treatment in IDDVT.

Schwarz et al. [34] compared I 0 days of LMWH with 3 months of compression therapy versus compression therapy alone. The study failed to show any superiority of this short duration LMWH therapy in reducing proximal extension or PE among the 'low-risk' patients with IDDVT. The CACTUS study was a randomized double-blind placebo-controlled study looking at standard anticoagulation for the same 'low-risk' patient population [35]. The study had to be prematurely ter- 
minated due to expiry of the study drug before reaching the desired level of recruitment and hence carries low predictive value. However, from the intention to treat analysis, LMWH (nadroparin) for 6 weeks was found to be not superior to placebo in preventing proximal clot extension or reducing the incidence of PE. The treatment arm was actually found to have significantly higher rates of major as well as non-major bleeding events; risk difference $4.1 \%(95 \% \mathrm{Cl} 0.4$ to 9.2 ; $\mathrm{p}=0.0255)$. In a comprehensive meta-analysis by Franco et al. [33], the authors reported a significantly lower risk of VTE recurrence as well as PE in those who were treated with LMWH or oral anti-coagulants compared to no treatment with serial DUS only. They also reported no significant increase in major bleeding episodes among those who underwent anti-coagulation.

The most recent Cochrane review (2020) by Kirkilesis et al. [36] looked at the evidence for anti-coagulation against no-treatment or placebo in IDDVT. It concluded that the overall rate of VTE and DVT recurrence was reduced in those who underwent anti-coagulation compared to those in the placebo group or no-treatment group. However, there was no clear advantage of anti-coagulation in terms of prevention of PE. As for the duration of anti-coagulation, there was no difference between groups treated for 6 weeks as opposed to 12 weeks. Similar to the analysis by Franco and colleagues, this also found no significant difference in major bleeding episodes with the anti-coagulation. However, there was an increased incidence of clinically relevant, non-major bleeding.

\section{Conclusions}

While therapeutic anti-coagulation remains the benchmark in management of PDVT, the place and need for anti-coagulation in IDDVT remain a clinical conundrum. IDDVT remains an extremely common clinical condition accounting to approximately $50 \%$ of all diagnosed patients with DVT. Nevertheless, the number of good quality prospective randomized trials studying the place of routine anti-coagulation in IDDVT are few. Furthermore, as shown above, findings from these available studies are conflicting and do not offer clear guidance in formulating a management protocol.

While some studies show a benefit in anti-coagulation for all patients detected with IDDVT, other studies concluded that such treatment did not show any conclusive benefit over no intervention and possibly carries a higher risk of clinically significant bleeding episodes. Given the uncertainty and the lack of quality data to offer conclusive evidence, the final decision lies with the treating clinician to decide on an individualized plan of management. Those with severe symptoms, recurrent DVT or considered 'high-risk' for thrombus extension would benefit by therapeutic anti-coagulation over serial DUS monitoring. On the contrary, a careful assessment of risk-benefit balance is required in the low-risk patient with minimal symptoms where the probable benefit of anti-coagulation has to be balanced against potential adverse effects such as clinically significant bleeding. Such low-risk patients with IDDVT are possibly best managed by serial DUS screening if the logistics for such screening and follow up are feasible. Possible treatment of such patients with prophylactic dose anti-coagulation rather than therapeutic doses is a possible trade-off between minimising the bleeding risk and achieving the desired anti-coagulant effect. However, such interventions have not yet been tested in large scale studies at present time.

\section{Conflict of interest}

None.

\section{References:}

I. Heit JA, Spencer FA, White RH. The epidemiology of venous thromboembolism. J Thromb Thrombolysis. 2016; 4I(I): 3-14, doi: |0. 1007/s | |239-0I 5- |3 | I-6, indexed in Pubmed: 26780736.

2. https://www cdc gov/ncbddd/dvt/data. Data and Statistics on Venous Thromboembolism | CDC.

3. Wei M, Zhu J, Yi X, et al. The prevalence of isolated calf deep vein thrombosis in patients with pulmonary embolism. Int Angiol. 2013; 32(5): 465-470, indexed in Pubmed: 23903304.

4. Mattos MA, Melendres G, Sumner DS, et al. Prevalence and distribution of calf vein thrombosis in patients with symptomatic deep venous thrombosis: a color-flow duplex study. J Vasc Surg. 1996; 24(5): 738-744, doi: 10.1016/s074|-52।4(96)70006-x, indexed in Pubmed: 8918317.

5. Sapp B, Craddock G, Sapp J. Patterns and Distribution of Deep Vein Thrombus in the Lower Extremity. Journal for Vascular Ultrasound. 2018; 39(2): 7I-77, doi: I0.1 I77/I5443I67/50390020I.

6. Wells PS, Ginsberg JS, Anderson DR, et al. Use of a clinical model for safe management of patients with suspected pulmonary embolism. Ann Intern Med. 1998; 129(12): 997-1005, doi: 10.7326/0003-4819-129-12-199812150-00002, indexed in Pubmed: 9867786.

7. Goodacre S, Sutton AJ, Sampson FC. Meta-analysis: The value of clinical assessment in the diagnosis of deep venous thrombosis. Ann Intern Med. 2005; 143(2): 129-139, doi: 10.7326/000348|9-|43-2-200507 I90-000|2, indexed in Pubmed: 16027455.

8. Sartori M, Cosmi B, Legnani C, et al. The Wells rule and D-dimer for the diagnosis of isolated distal deep vein thrombosis. J Thromb Haemost. 2012; 10(II): 2264-2269, doi: 10.1 III/j.1538-7836.20|2.04895.x, indexed in Pubmed: 22906051

9. Di Nisio M, Squizzato A, Rutjes AWS, et al. Diagnostic accuracy of D-dimer test for exclusion of venous thromboembolism: a systematic review. J Thromb Haemost. 2007; 5(2): 296-304, doi: I0.1 I I I/j.1538-7836.2007.02328.x, indexed in Pubmed: I7|55963. 
10. Goodacre S, Sampson F, Thomas S, et al. Systematic review and meta-analysis of the diagnostic accuracy of ultrasonography for deep vein thrombosis. BMC Med Imaging. 2005; 5: 6, doi: 10.1 I86/I47I-2342-5-6, indexed in Pubmed: 16202I35.

II. Zhang Y, Xia H, Wang Y, et al. The rate of missed diagnosis of lower-limb DVT by ultrasound amounts to $50 \%$ or so in patients without symptoms of DVT: A meta-analysis. Medicine (Baltimore). 2019; 98(37): el7103, doi: 10.1097/ MD. 0000000000017103 , indexed in Pubmed: 315I784I.

12. Heit J, Petterson T, Farmer S, et al. Trends in the incidence of deep vein thrombosis and pulmonary embolism: a 35-year population-based study. Blood. 2006; I08(II): |488-| 488, doi: 10.1 182/blood.v108.II.1488.1488.

13. Needleman L, Cronan J, Lilly M, et al. Ultrasound for lower extremity deep venous thrombosis. Circulation. 2018; 137(14): 1505-1515, doi: 10.1 161/circulationaha. I 17.030687.

14. Kearon C, Kearon C. Natural history of venous thromboembolism. Circulation. 2003; 107(23 Suppl I): 122-130, doi: I0.1I6I/0I.CIR.0000078464.8267I.78, indexed in Pubmed: $128 \mid 4982$.

15. Sevestre MA, Quashié C, Genty C, et al. Clinical presentation and mortality in pulmonary embolism: The Optimev study. Journal des Maladies Vasculaires. 2010; 35(4): 242-249, doi: 10.1016/j.jmv.2010.05.004.

16. Galanaud JP, Quenet S, Rivron-Guillot K, et al. RIETE INVESTIGATORS. Comparison of the clinical history of symptomatic isolated distal deep-vein thrombosis vs. proximal deep vein thrombosis in II 086 patients. J Thromb Haemost. 2009; 7(I2): 2028-2034, doi: 10.1 I I I/j.1538-7836.2009.03629.x, indexed in Pubmed: 19793188.

17. Fleck $D$, Albadawi $H$, Wallace $A$, et al. Below-knee deep vein thrombosis (DVT): diagnostic and treatment patterns. Cardiovasc Diagn Ther. 2017; 7(Suppl 3): S134-S139, doi: 10.21037/ cdt.2017.1 I.03, indexed in Pubmed: 29399516.

18. Garry J, Duke A, Labropoulos N. Systematic review of the complications following isolated calf deep vein thrombosis. $\mathrm{Br} J$ Surg. 2016; 103(7): 789-796, doi: 10.1002/bjs.10152, indexed in Pubmed: 27060255.

19. Kearon C, Akl EA, Ornelas J, et al. Antithrombotic therapy for VTE disease: CHEST guideline and expert panel report. Chest. 2016; 149(2): 315-352, doi: 10.1016/j.chest.2015.11.026, indexed in Pubmed: 26867832.

20. Galanaud JP, Sevestre MA, Genty C, et al. OPTIMEV-SFMV investigators. Incidence and predictors of venous thromboembolism recurrence after a first isolated distal deep vein thrombosis. J Thromb Haemost. 2014; 12(4): 436-443, doi: 10.1 II I/ jth. I25 I2, indexed in Pubmed: 24450376.

21. Sartori M, Migliaccio L, Favaretto E, et al. Two years outcome of isolated distal deep vein thrombosis. Thromb Res. 2014; 134(I): 36-40, doi: 10.1016/j.thromres.2014.03.033, indexed in Pubmed: 247। 3107.

22. Barco S, Corti M, Trinchero A, et al. Survival and recurrent venous thromboembolism in patients with first proximal or isolated distal deep vein thrombosis and no pulmonary embolism. J Thromb Haemost. 2017; 15(7): 1436-1442, doi: 10.1। II/ jth. I37I3, indexed in Pubmed: 28439954.

23. Galanaud JP, Monreal M, Kahn SR, et al. Epidemiology of the post-thrombotic syndrome. Thromb Res. 2018(164): 100-109.
24. Meissner MH, Caps MT, Bergelin RO, et al. Early outcome after isolated calf vein thrombosis. J Vasc Surg. 1997; 26(5): 749-756, doi: 10.1016/s0741-5214(97)70086-7, indexed in Pubmed: $93728 \mathrm{I}$ I.

25. Saarinen JP, Domonyi K, Zeitlin R, et al. Postthrombotic syndrome after isolated calf deep venous thrombosis: the role of popliteal reflux. J Vasc Surg. 2002; 36(5): 959-964, doi: 10.1067/ mva.2002. 127523, indexed in Pubmed: 12422091.

26. McLafferty RB, Moneta GL, Passman MA, et al. Late clinical and hemodynamic sequelae of isolated calf vein thrombosis. J Vasc Surg. 1998; 27(I): 50-56; discussion 56, doi: 10.1016/s07415214(98)7029I-5, indexed in Pubmed: 9474082.

27. Galanaud JP, Righini M, Le Collen L, et al. Long-term risk of postthrombotic syndrome after symptomatic distal deep vein thrombosis: The CACTUS-PTS study. J Thromb Haemost. 2020; 18(4): 857-864, doi: 10.11 II/jth.14728, indexed in Pubmed: 31899848.

28. Kearon C, Kahn SR, Agnelli G, et al. Antithrombotic therapy for venous thromboembolic disease: American College of Chest Physicians Evidence-Based Clinical Practice Guidelines (8th Edition). Chest. 2008; 133 (6 Suppl): 454S-545S, doi: 10.1378/ chest.08-0658, indexed in Pubmed: 18574272.

29. Kahn SR, Shapiro S, Wells PS, et al. SOX trial investigators. Compression stockings to prevent post-thrombotic syndrome: a randomised placebo-controlled trial. Lancet. 2014; 383(9920): 880-888, doi: 10. I016/SO 140-6736(I3)61902-9, indexed in Pubmed: 24315521.

30. Palareti G. How I Treat isolated distal DVT. Blood. 2015; 123: 1802-1810.

31. Pinede L, Ninet J, Duhaut $P$, et al. Investigators of the "Durée Optimale du Traitement AntiVitamines K" (DOTAVK) Study. Comparison of 3 and 6 months of oral anticoagulant therapy after a first episode of proximal deep vein thrombosis or pulmonary embolism and comparison of 6 and 12 weeks of therapy after isolated calf deep vein thrombosis. Circulation. 200I; 103(20): 2453-2460, doi: 10.1 I6I/0I.cir.103.20.2453, indexed in Pubmed: I 1369685.

32. Ferrara F, Meli F, Amato $C$, et al. Optimal duration of treatment in surgical patients with calf venous thrombosis involving one or more veins. Angiology. 2006; 57(4): 418-423, doi: 10.11 177/00033 19706290745 , indexed in Pubmed: 17022376.

33. Franco L, Giustozzi M, Agnelli G, et al. Anticoagulation in patients with isolated distal deep vein thrombosis: a meta-analysis. J Thromb Haemost. 2017; 15(6): II42-II54, doi: 10.1III/ jth. 13677 , indexed in Pubmed: 28316I24.

34. Schwarz T, Buschmann L, Beyer J, et al. Therapy of isolated calf muscle vein thrombosis: a randomized, controlled study. J Vasc Surg. 2010; 52(5): 1246-1250, doi: 10.1016/j.jvs.2010.05.094, indexed in Pubmed: 20630682.

35. Righini M, Galanaud JP, Guenneguez H, et al. Anticoagulant therapy for symptomatic calf deep vein thrombosis (CACTUS): a randomised, double-blind, placebo-controlled trial. Lancet Haematol. 2016; 3(I2): e556-e562, doi: 10.1016/\$23523026(I6)30I3I-4, indexed in Pubmed: 27836513.

36. Kirkilesis G, Kakkos SK, Bicknell C, et al. Treatment of distal deep vein thrombosis. Cochrane Database Syst Rev. 2020; 4: CD013422, doi: 10.1002/I465I858.CD013422.pub2, indexed in Pubmed: 32271939. 